\title{
Zero Index Metamaterial for Designing High-Gain Patch Antenna
}

\author{
Yahong Liu, Xiaojing Guo, Shuai Gu, and Xiaopeng Zhao \\ Smart Materials Laboratory, Department of Applied Physics, Chang'an Campus, Northwestern Polytechnical University, \\ Xian 710129, China \\ Correspondence should be addressed to Xiaopeng Zhao; xpzhao@nwpu.edu.cn
}

Received 16 April 2013; Revised 12 August 2013; Accepted 14 August 2013

Academic Editor: Alistair P. Duffy

Copyright (C) 2013 Yahong Liu et al. This is an open access article distributed under the Creative Commons Attribution License, which permits unrestricted use, distribution, and reproduction in any medium, provided the original work is properly cited.

A planar wideband zero-index metamaterial (ZIM) based on mesh grid structure is studied. It is demonstrated that the real part of the index approaches zero at the wideband covering from $9.9 \mathrm{GHz}$ to $11.4 \mathrm{GHz}$. Two conventional patch antennas whose operating frequencies are both in the range of zero-index frequencies are designed and fabricated. And then, the ZIM is placed in the presence of the conventional patch antennas to form the proposed antennas. The distance between the antenna and the ZIM cover is investigated. Antenna performances are studied with simulations and measurements. The results show that the more directional and higher gain patch antennas can be obtained. The measured results are in good agreement with the simulations. Compared to the conventional patch antenna without the ZIM, it is shown that the beamwidth of antenna with the ZIM cover becomes more convergent and the gain is much higher.

\section{Introduction}

In recent years, artificial electromagnetic metamaterials have attracted growing interests. Based on the effective medium theory, electromagnetic metamaterials can be characterized by electric permittivity and magnetic permeability. Pendry realized the artificially electric plasma using a metallic wire whose permittivity is negative [1]. And then, Pendry discovered the artificially magnetic plasma whose permeability is negative [2]. Metamaterials open a door to realize all possible material properties by designing different cellular architectures and using different substrate materials [3-6]. Among the various, unusual material parameters provided by metamaterials, zero permittivity/permeability/index is a singular material parameter, which can lead to many interesting phenomena and applications [7-22]. Jiang et al. [7] and Jin and $\mathrm{He}$ [8] demonstrated that effective zero index metamaterial (ZIM) can enhance uniform fields. Silveirinha et al. [9-12] proposed that the electromagnetic wave can tunnel through a zero electric permittivity metamaterial. Nguyen et al. [13] and Hao et al. [14] investigated that ZIM with defects can realize the total transmission or reflection of the impinging electromagnetic wave. Therefore, ZIM structure can offer advances in shielding or cloaking technologies without restricting the object's viewpoint.

Recent studies show that ZIM may have paved a new way for designing novel high-gain antennas due to its unique properties. Based on Snell's law, it is considered an incident ray on an interface of the ZIM with grazing incidence that comes from a source inside ZIM. A near-zero index ray in the media will be refracted in a direction that is very close to the normal. The lower the optical index is, the closer the normal direction is. Enoch et al. [15] is the first to realize high directive radiation by employing monopole source embedded in ZIM, thus confining the radiated energy to a small solid angle. After the work of Enoch, Wu et al. [16] proposed a lefthanded metamaterial as a substrate for designing directional radiation. Through the control of the structure's geometry, the zero index frequency can be tuned to the desired specification to produce directional emission. In addition, other works about directive radiations employing ZIM were also studied [17-19]. However, in the previous references, the directive antennas based on ZIM operate at a single frequency or a narrowband frequency. 
Inspired by Enoch, in this paper, a planar wideband ZIM is fabricated firstly. The resonant electromagnetic properties present in the bandwidth of the planar ZIM can be up to 1.5 GHz. And then, two high-gain patch antennas (narrowband patch antenna and wideband patch antenna) based on the ZIM cover are studied. The antennas' performances are studied with simulations and measurements. It is demonstrated that gain and directivity of the proposed antennas can be improved at the wideband frequencies compared to the conventional patch antennas without the ZIM. In addition, the electric field distributions are presented for explaining physically the improvement of antenna performance. A simple method for achieving a wideband high-gain patch antenna is provided in the present work.

\section{Zero Index Metamaterial}

2.1. ZIM Design Principle. Pendry discovered that electromagnetic behaviors of arrays of periodical wires are similar to those of metal [1]. Plasma is a system composed of a large number of charged particles, which shows neutral. The effective permittivity can be expressed as

$$
\varepsilon(\omega)=1-\frac{\omega_{p}^{2}}{\omega^{2}},
$$

where $\omega_{p}$ is the plasma frequency and $\omega$ is the frequency of the propagating electromagnetic wave. The plasma frequency $\omega_{p}$ of the metal can be expressed as

$$
\omega_{p}^{2}=\frac{n_{e} e^{2}}{\varepsilon_{0} m_{\mathrm{eff}}},
$$

where $n_{e}$ is the charge density, $e$ is the electric quantity, $m_{\mathrm{eff}}$ is the effective mass, and $\varepsilon_{0}$ is the permittivity in free space. The plasma frequency $\omega_{p}$ of the metal is in the frequency of ultraviolet. Pendry proposed a mechanism for the depression of the plasma frequency $\omega_{p}$ to microwave by employing arrays of wires. Wires can depress $n_{e}$ and increase $m_{\text {eff }}$, resulting in lowering the plasma frequency $\omega_{p}$. The plasma frequency $\omega_{p}$ of the wires can be expressed as

$$
\omega_{p}^{2}=\frac{2 \pi c_{0}^{2}}{a^{2} \ln (a / r)},
$$

where $r$ is the radius of the wires, $a$ is the lattice constant, and $c_{0}$ is the speed of light in the free space. From (3), the plasma frequency $\omega_{p}$ can be lowered by optimizing the lattice constant $a$ and the radius $r$ of the wires. It can be concluded that the permittivity is negative when the frequency is below the plasma frequency.

Based on Pendry's thought, we design the mesh grid structure whose plasma frequency can be in the microwave band by optimizing the parameters of the mesh grid structure. When operating at the plasma frequency, the effective permittivity is zero, and hence it yields a zero index.

2.2. Fabrication and Experiment. Figure 1 shows the planar ZIM consisting of arrays of mesh grid on each side of the substrate with the thickness of $1.5 \mathrm{~mm}$ and the effective dielectric constant $\varepsilon_{r}$ of $2.65(\operatorname{tg} \theta=0.009)$. The design idea is inspired by [15]. However, in the present paper, we utilize single-layer microstrip technology for realizing the planar ZIM. Geometrical dimension of the unit cell presented in Figure 1(a) are line width $w$ and lattice constant $l$. Zero index frequency can be controlled by constructing the parameters $w$ and $l$. In the present paper, the simulations were employed by using German commercial software package CST Microwave Studio on the basis of the finite integration method. Electromagnetic resonant behaviors of the planar ZIM for different parameters $w$ and $l$ are studied. Figure 2 shows the resonant behaviors for $w=0.2 \mathrm{~mm}, 0.4 \mathrm{~mm}, 06 \mathrm{~mm}$, and $0.8 \mathrm{~mm}$ with constant $l=14 \mathrm{~mm}$. Figures 2(a) and 2(b) show the transmission spectrums and reflection spectrums. The calculated permeability, permittivity, and index by using $S$-parameters retrieval method [23] are presented in Figures 2(c), 2(d), 2(e), 2(f), 2(g), and 2(h). It is presented that the cutoff nearzero index is $9.9 \mathrm{GHz}, 10.9 \mathrm{GHz}, 11.7 \mathrm{GHz}$, and $12.39 \mathrm{GHz}$ corresponding to $w=0.2 \mathrm{~mm}, 0.4 \mathrm{~mm}, 06 \mathrm{~mm}$, and $0.8 \mathrm{~mm}$. The near-zero index shifts the higher frequency with the increase of the parameter $w$. Meanwhile, the results show that the imaginary parts of the permeability, permittivity, and index are all small at the near-zero index. The electromagnetic behaviors of the planar ZIM versus the parameter $l$ are also investigated. The study shows that the near-zero index shifts the lower frequency with the increase of $l$ (not shown here).

The planar ZIM structure was fabricated by using a shadow mask/etching technique in this paper. The geometrical dimensions of the unit cell are chosen as follows: line width $w=0.6 \mathrm{~mm}$ and lattice constant $l=14 \mathrm{~mm}$. Deposited copper thickness is $35 \mu \mathrm{m}$. The fabricated planar ZIM sample is shown in Figure 1(b). The experiments composed of two standard horn antennas $(8.2-12.4 \mathrm{GHz})$ were carried out with an AV3618 network analyzer $(50 \mathrm{MHz}-20 \mathrm{GHz})$ in an anechoic chamber. The measured refractive index is shown in Figure 2(g), where the real part of the index is near-zero at the wideband frequencies.

\section{High-Gain Patch Antennas with the ZIM Cover}

3.1. Antennas' Design and Fabrication. Based on the wideband zero index, besides a conventional narrowband patch antenna, a wideband patch antenna has also been designed and fabricated. Antennas' design is employed by CST Microwave Studio and the fabrication is by using a shadow mask/etching technique on the $1.5 \mathrm{~mm}$ thickness substrate with an effective dielectric constant $\varepsilon_{r}$ of $2.65(\operatorname{tg} \theta=0.009)$. The dimension of the radiation patch is $9.7 \mathrm{~mm} \times 7.8 \mathrm{~mm}$. The ground plane size and substrate size are both $56 \mathrm{~mm} \times 56 \mathrm{~mm}$. A $50 \Omega$ coaxial probe which is used to feed the antenna was situated at the centre of a rectangular patch along the $y$-axis, and $2.3 \mathrm{~mm}$ away from the $x$-axis in the cartesian coordinate. The fabricated narrowband antenna sample is shown in Figure 3(a). The simulated and measured antenna reflections are presented in Figure 3(c). It shows that the simulated $-10 \mathrm{~dB}$ bandwidth is $0.846 \mathrm{GHz}$ covering 


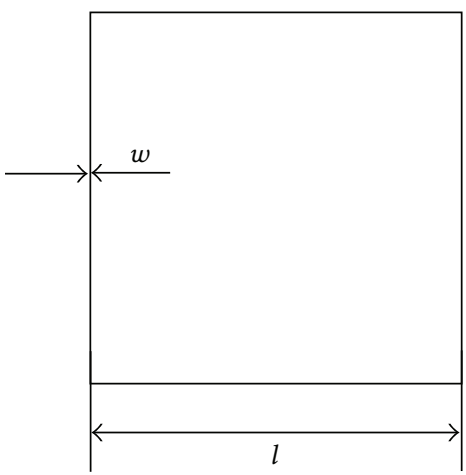

(a)



(b)

FIgure 1: Zero index metamaterial, (a) the unit cell and (b) the sample.

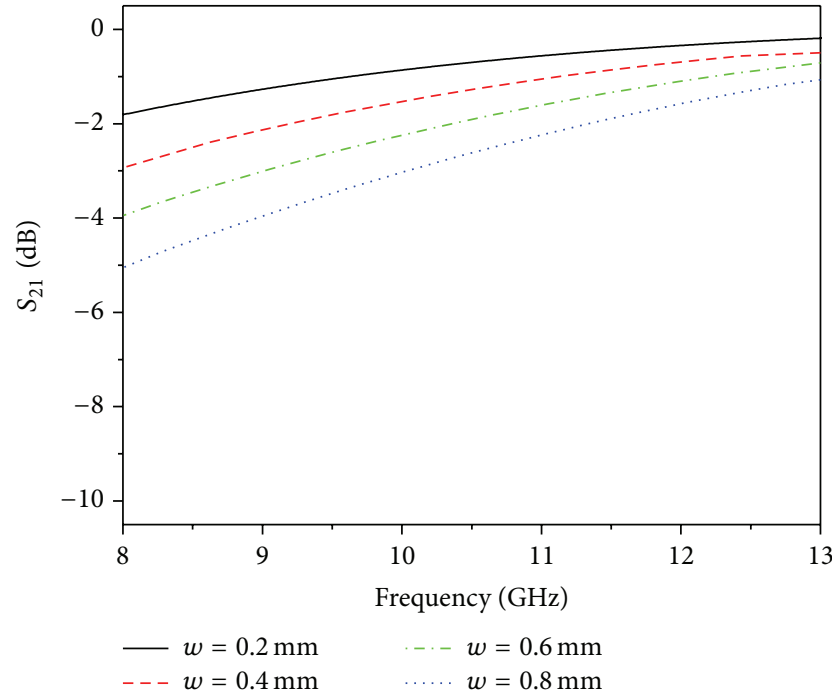

(a)

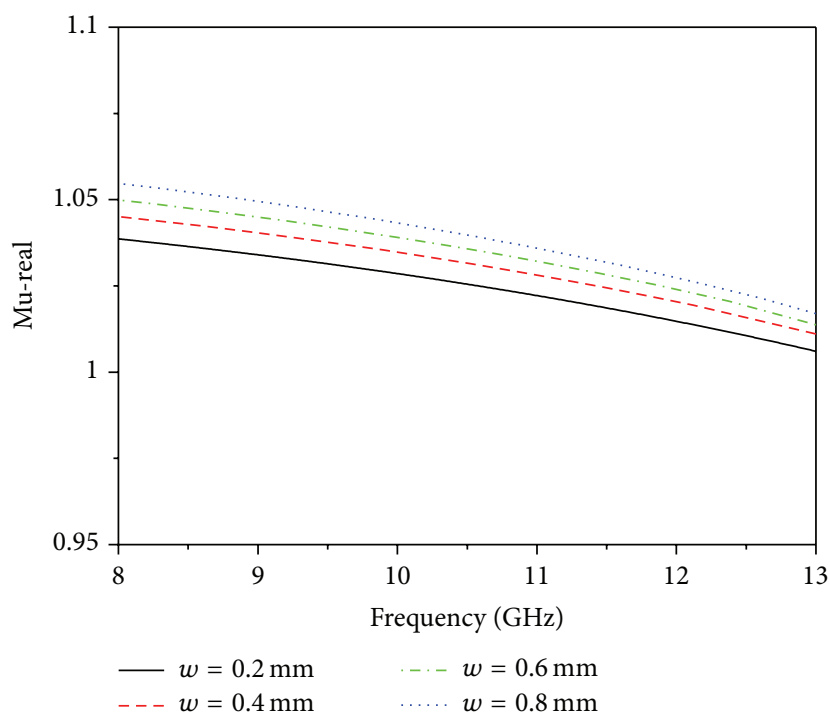

(c)

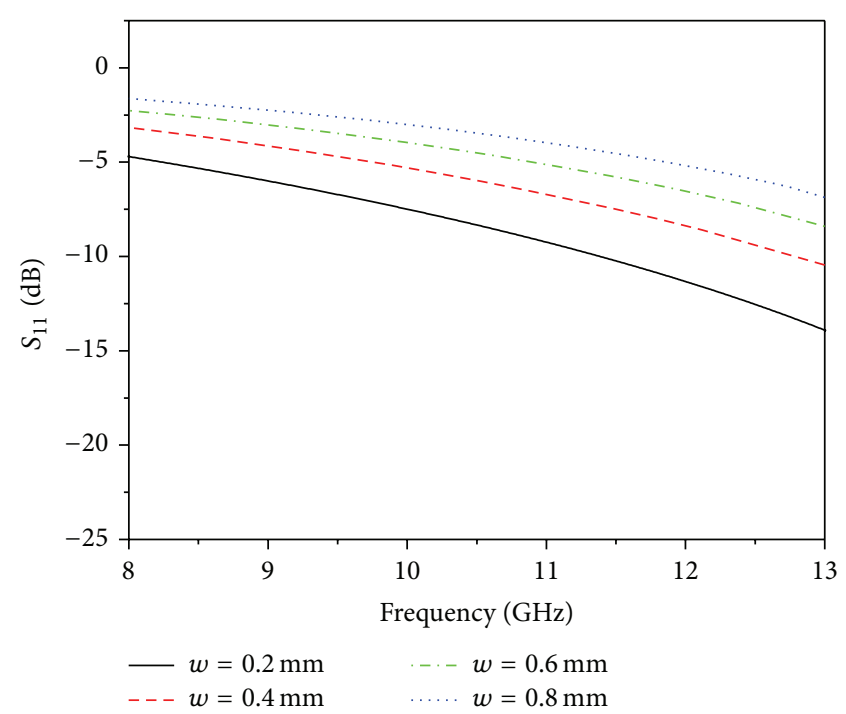

(b)

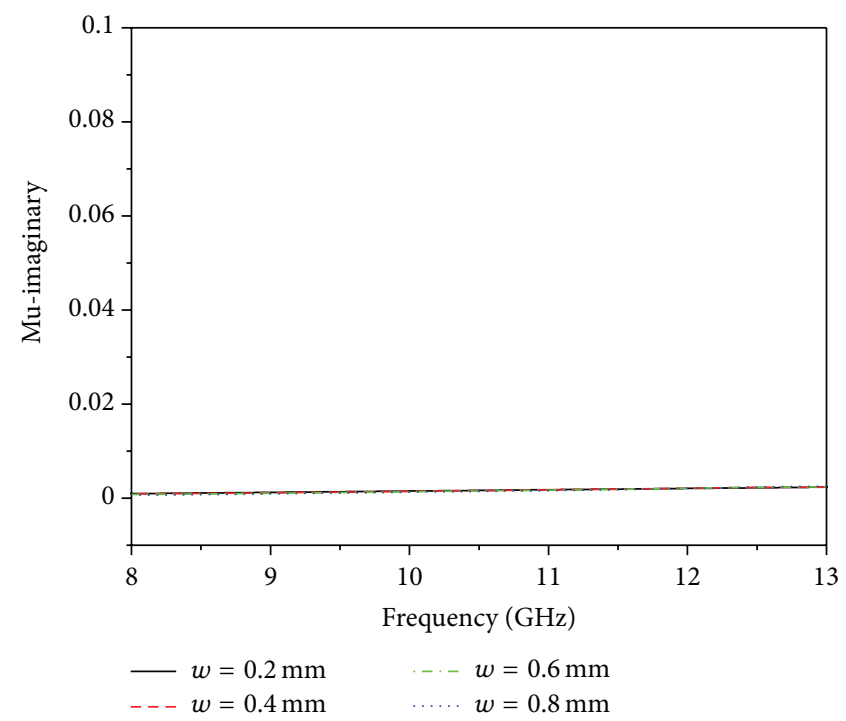

(d)

Figure 2: Continued. 


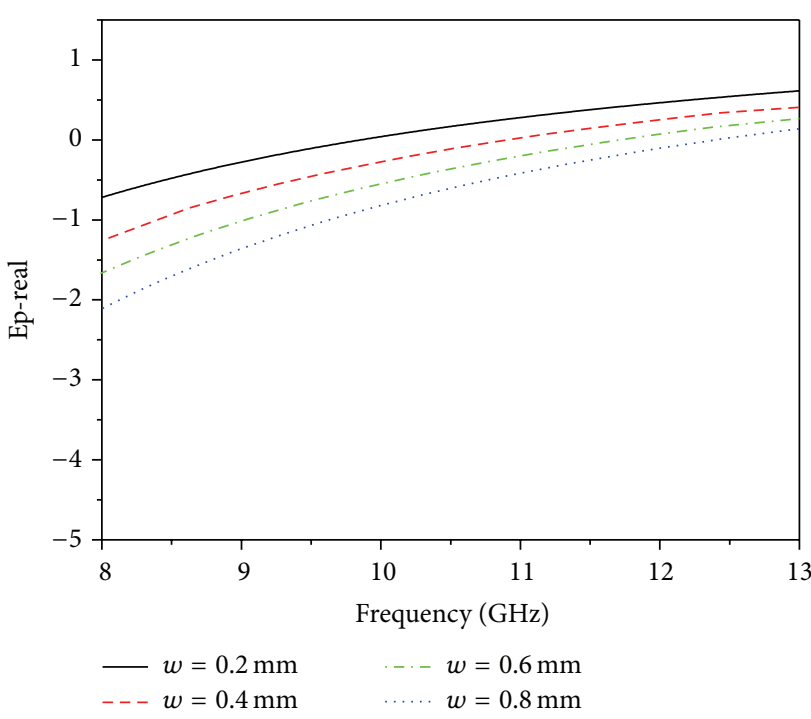

(e)

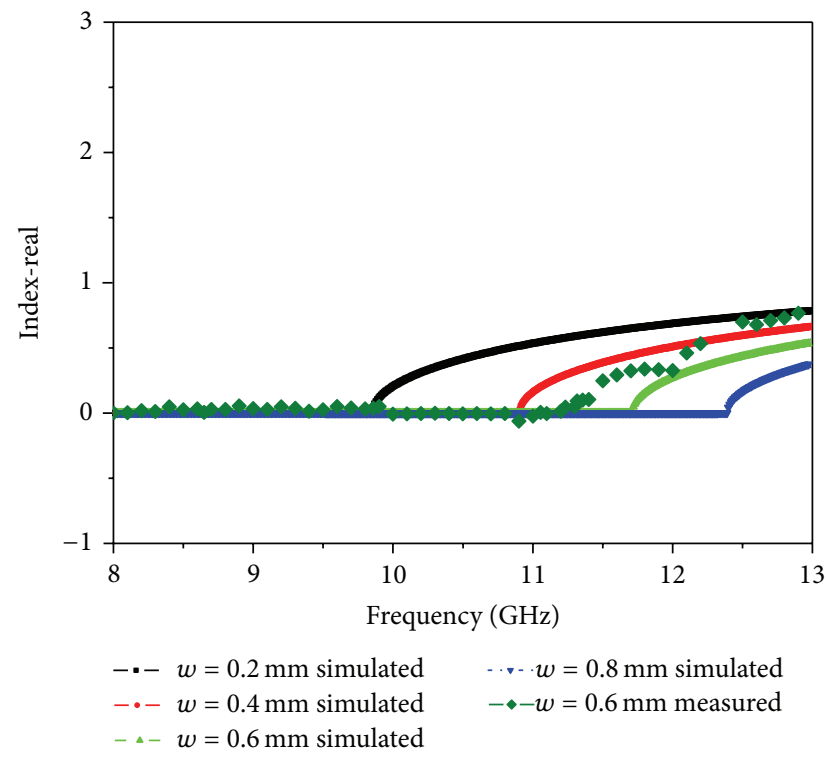

(g)

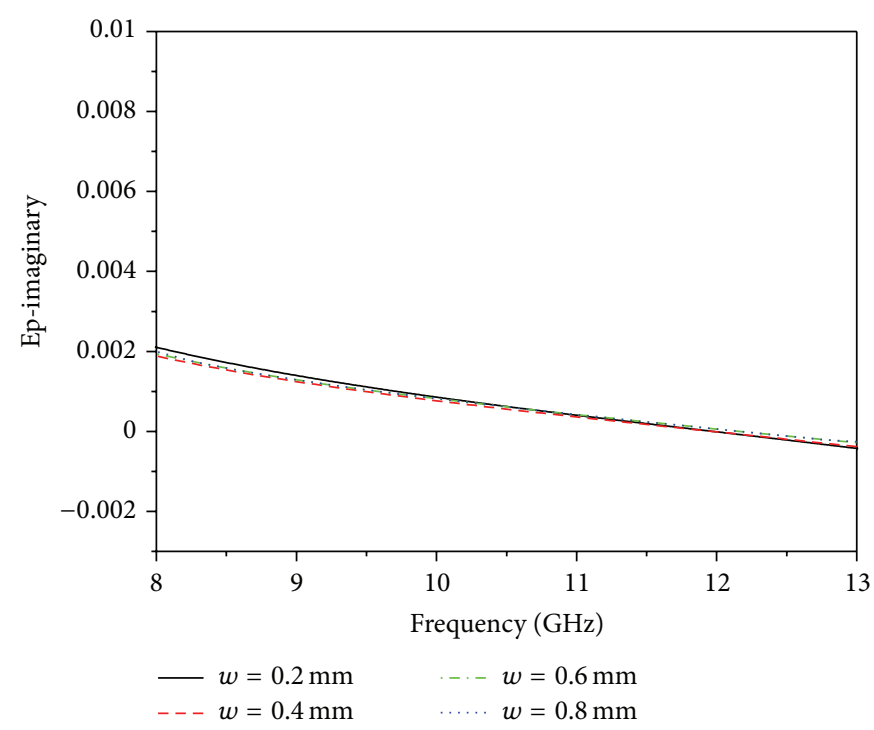

(f)

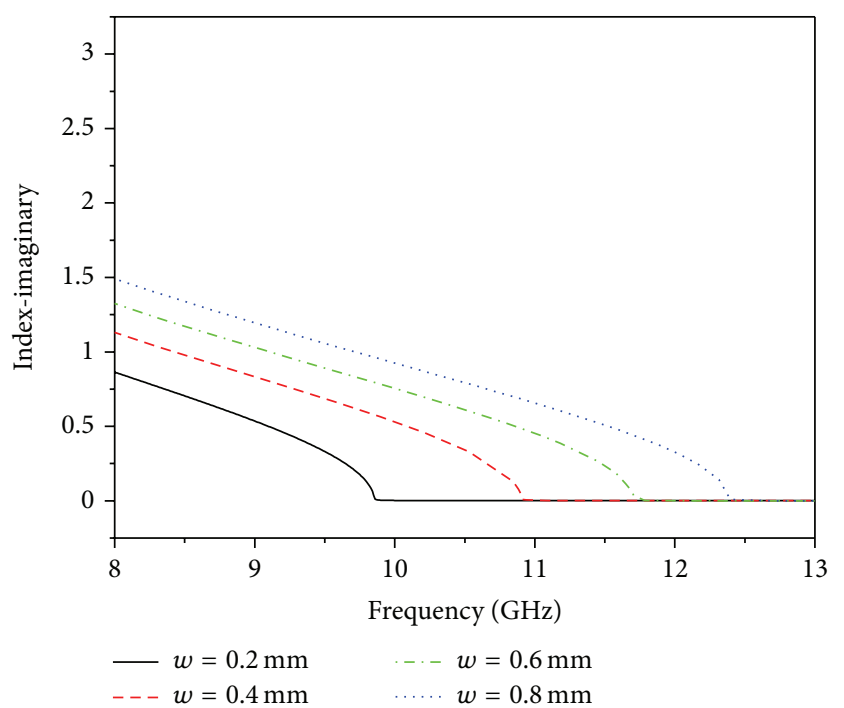

(h)

FIGURE 2: The results for $w=0.2 \mathrm{~mm}, 0.4 \mathrm{~mm}, 0.6 \mathrm{~mm}$, and $0.8 \mathrm{~mm}$ with $l=14 \mathrm{~mm}$, (a) the simulated transmission spectrums, (b) the simulated reflection spectrums, (c) the simulated real parts of the permeability, (d) the simulated imaginary parts of the permeability, (e) the simulated real parts of the permittivity, (f) the simulated imaginary parts of the permittivity, (g) the simulated and measured real parts of the index, and (h) the simulated imaginary parts of the index.

from $9.914 \mathrm{GHz}$ to $10.76 \mathrm{GHz}$, with the relative bandwidth of $8.184 \%$. Whereas the measured $-10 \mathrm{~dB}$ bandwidth is $0.8 \mathrm{GHz}$ covering from $10.14 \mathrm{GHz}$ to $10.94 \mathrm{GHz}$, with the relative bandwidth of $7.6 \%$. The measured frequency is slightly higher than the simulated one. This discrepancy may be due to the fabrication tolerance and the substrate material where the actual dielectric constant is a little different from the value used in the simulations.

In order to broaden antenna bandwidth, four parasitic patches [24] with the dimension of $2.3 \mathrm{~mm} \times 7.8 \mathrm{~mm}$ surrounded by the radiation patch are added. The wideband patch antenna prototype is shown in Figure 3(b). The simulated $-10 \mathrm{~dB}$ bandwidth is $1.212 \mathrm{GHz}$ covering from $9.884 \mathrm{GHz}$ to $11.096 \mathrm{GHz}$, with the relative bandwidth of $11.55 \%$. Whereas the measured $-10 \mathrm{~dB}$ bandwidth is $1.2 \mathrm{GHz}$ covering from $10.05 \mathrm{GHz}$ to $11.25 \mathrm{GHz}$, with the relative bandwidth of $11.3 \%$. The antenna bandwidth is wider by $0.4 \mathrm{GHz}$ than that of the narrowband patch antenna. The operation frequency of the wideband antenna is still in the range of zero index of the ZIM.

The prototype of the proposed antenna with the ZIM cover is shown in Figure 4. It is demonstrated that 


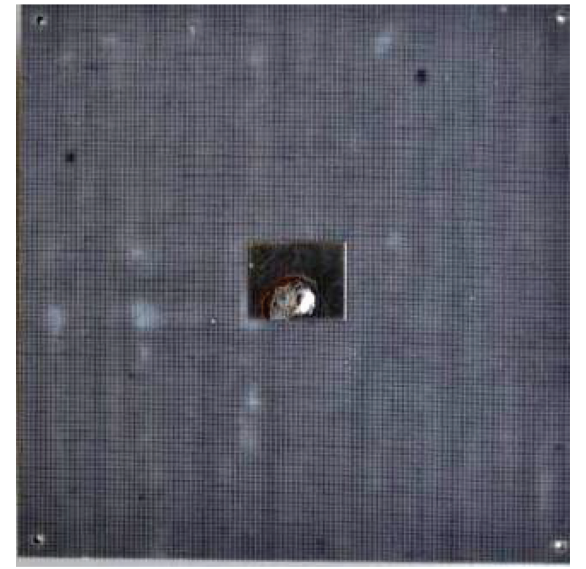

(a)

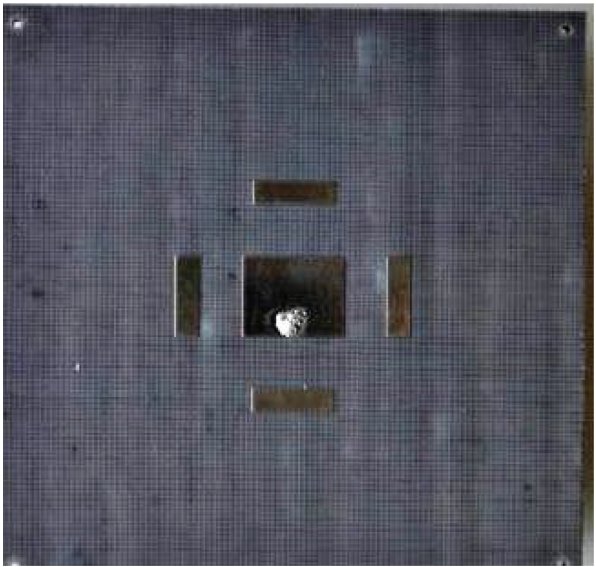

(b)

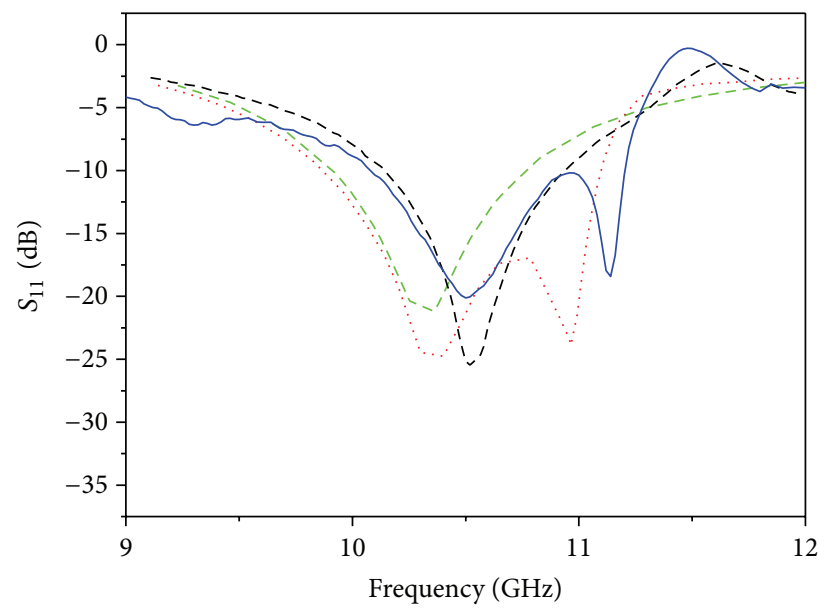

- - - Simulated conventional narrowband antenna

Simulated conventional wideband antenna

- - - Measured conventional narrowband antenna

_ Measured conventional wideband antenna

(c)

FIGURE 3: The conventional patch antennas, (a) the prototype of the conventional narrowband antenna, (b) the prototype of the conventional wideband antenna, and (c) the reflection coefficients.

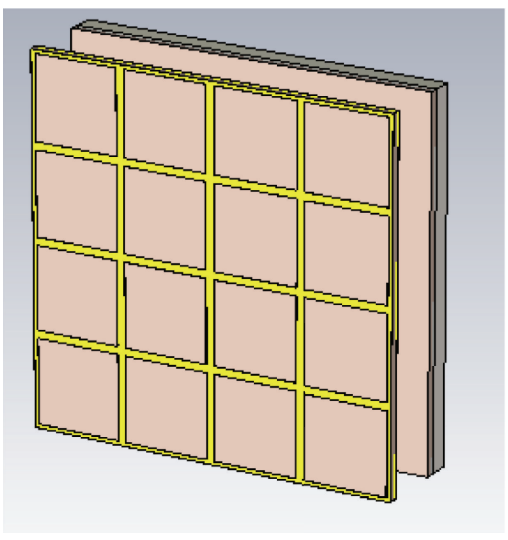

(a)

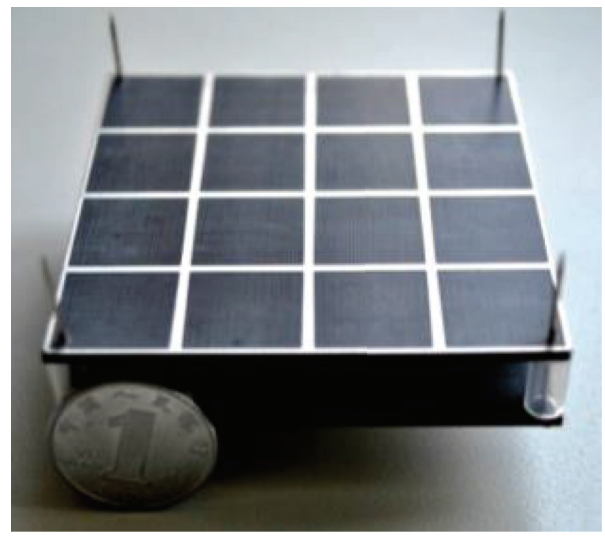

(b)

FIgURE 4: The prototype of the proposed wideband antenna with the ZIM cover, (a) the setup for the numerical simulations and (b) the proposed antenna sample. 




(a)

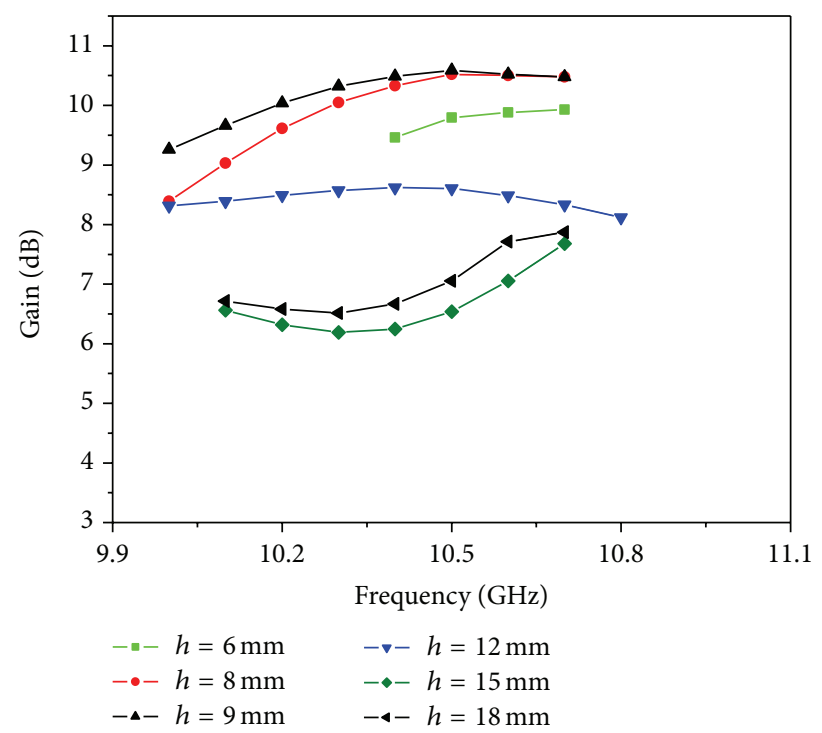

(b)

FIGURE 5: The performance of the proposed narrowband antenna with the ZIM cover for different distances $h$, (a) the reflection coefficients and (b) the antenna gains.

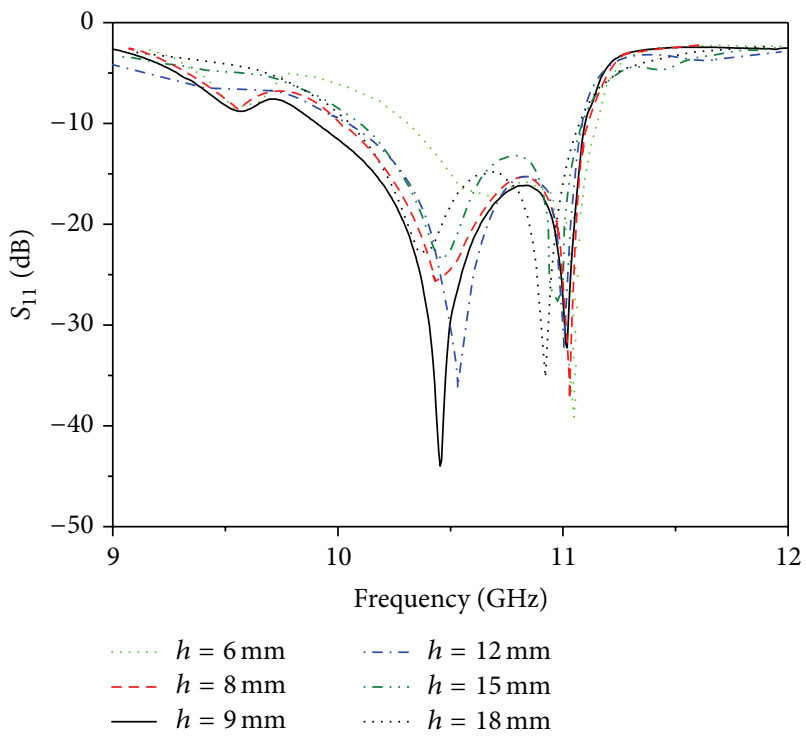

(a)

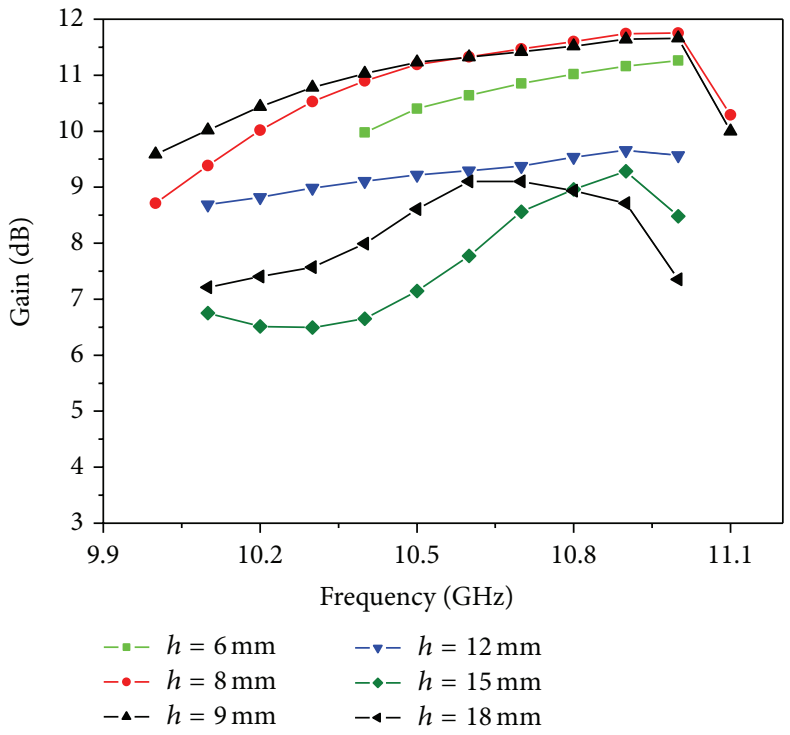

(b)

FIGURE 6: The performance of the proposed wideband antenna with the ZIM cover for different distances $h$, (a) the reflection coefficients and (b) the antenna gains.

the distance $h$ between the patch antenna and ZIM cover influences the antenna performances. Figure 5 gives the simulated performances of the narrowband patch antenna with the ZIM cover, which presents the optimum distance $h=9 \mathrm{~mm}$. The average gain of the narrowband antenna with the ZIM is $10.17 \mathrm{~dB}$ at the working frequencies, and the peak gain can be up to $10.59 \mathrm{~dB}$ at $10.5 \mathrm{GHz}$. Figure 6 gives the simulated performances of the wideband antenna with the ZIM cover. The optimum distance is also $h=9 \mathrm{~mm}$. The average gain of the proposed wideband antenna with
ZIM is $10.9 \mathrm{~dB}$ at the working frequencies, and the peak gain is $11.6 \mathrm{~dB}$ at $10.9 \mathrm{GHz}$. Therefore, in the present paper, we fabricate the antenna samples with the optimum distance $h=9 \mathrm{~mm}$. In addition, the number of the ZIM layers versus antenna performances is also investigated. The antenna performances are listed in Table 1. The results show that the antenna gain is improved with the increase of the ZIM layers. When one layer ZIM is placed above the conventional patch antenna, antenna beamwidth is convergent and the gain is improved greatly. When two layers ZIM or much more layers 


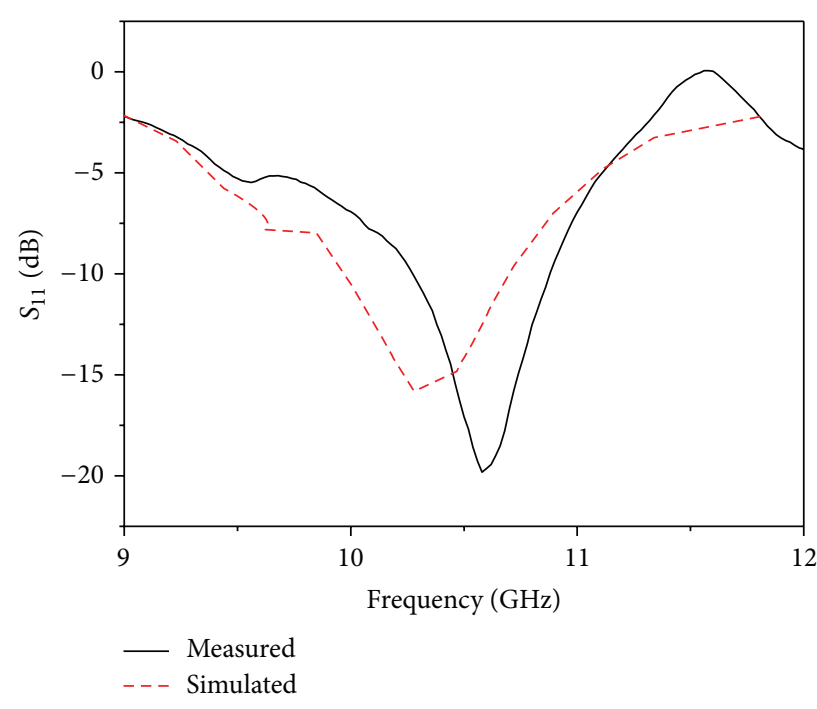

(a)

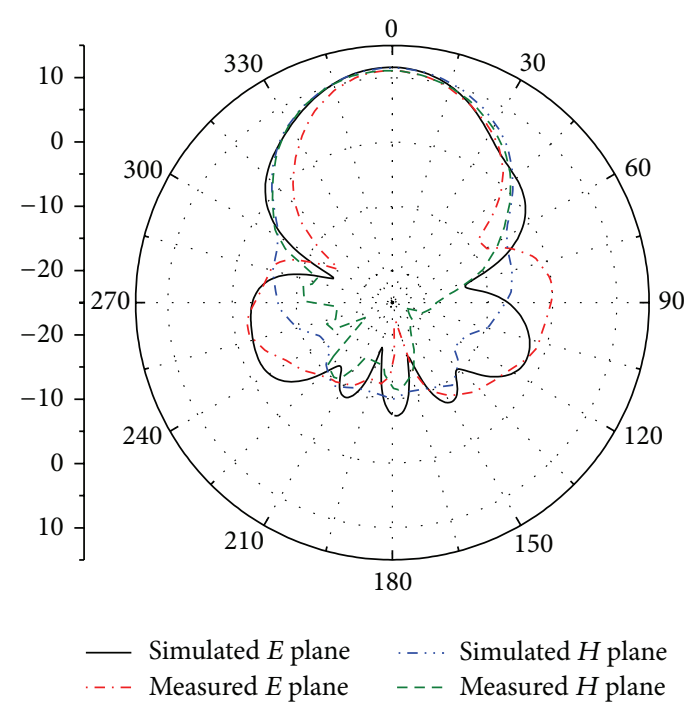

(b)

FIGURE 7: The performances of the proposed narrowband antenna with the ZIM cover, (a) the reflection coefficients and (b) the radiation patterns.

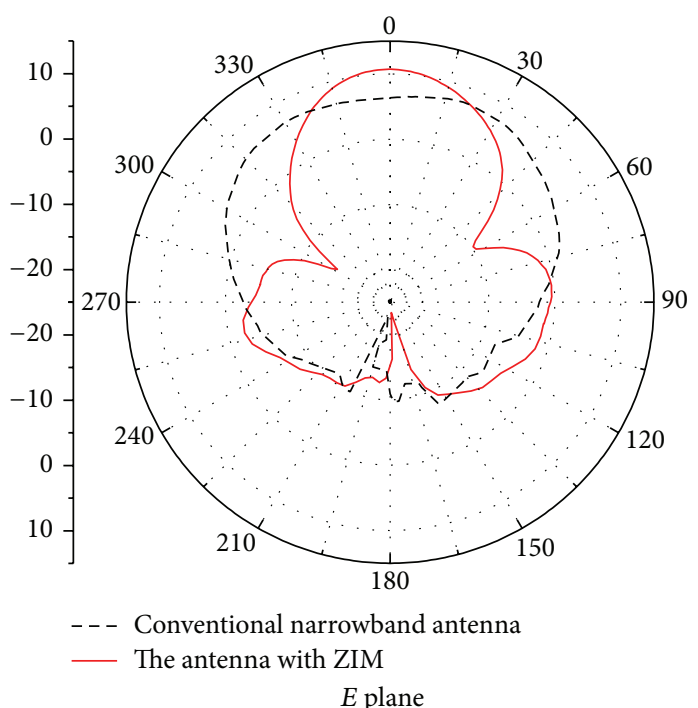

(a)

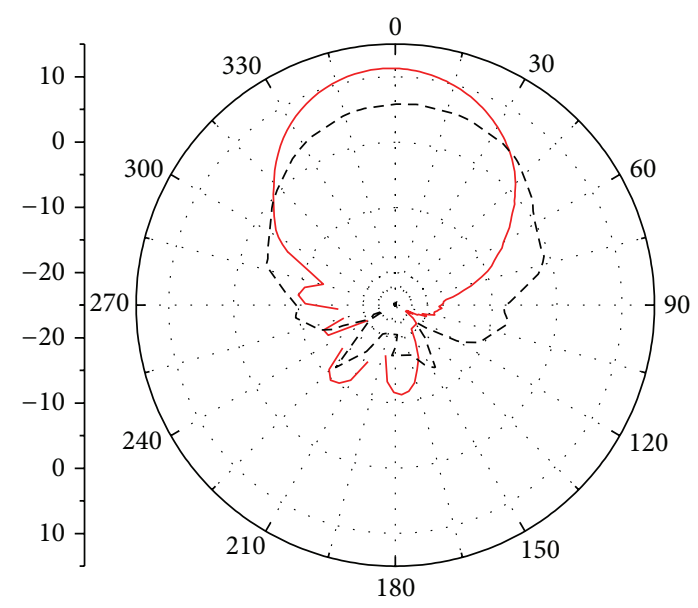

- - - Conventional narrowband antenna

The antenna with ZIM

$H$ plane

(b)

FIGURE 8: The measured radiation patterns for the conventional narrowband antenna and the antenna with the ZIM cover.

ZIM are utilized, antenna gain is improved slowly. When the ZIM cover is increased to seven layers, the antenna gain is almost stable. In order to design the antenna with compact volume and improved gain, the antenna based on one layer ZIM with the optimum distance $h=9 \mathrm{~mm}$ is fabricated in the present paper.

3.2. Performances of the Narrowband Patch Antenna with the ZIM Cover. Figure 7 presents the performances of the proposed narrowband high-gain antenna. The simulated and measured antenna reflection coefficients are shown in Figure $7(\mathrm{a})$, where the simulated $-10 \mathrm{~dB}$ bandwidth is
$0.71 \mathrm{GHz}$ covering from $9.98 \mathrm{GHz}$ to $10.69 \mathrm{GHz}$ and the measured $-10 \mathrm{~dB}$ bandwidth is $0.63 \mathrm{GHz}$ covering from $10.26 \mathrm{GHz}$ to $10.89 \mathrm{GHz}$. The proposed antenna radiation patterns are shown in Figure 7(b). It shows that the simulated half-power beamwidth (HPBW) in the $E$ plane and $H$ plane are $45^{\circ}$ and $51^{\circ}$, respectively. The measured HPBW in the $E$ plane and $H$ plane are $41^{\circ}$ and $49^{\circ}$, respectively. The simulated gain is $10.48 \mathrm{~dB}$ and the measured one by using the gain comparison method is $10.6 \mathrm{~dB}$. The simulated and measured aperture efficiencies are $24 \%$ and $23.7 \%$, respectively. The measured results are in good agreement with the simulated ones. 


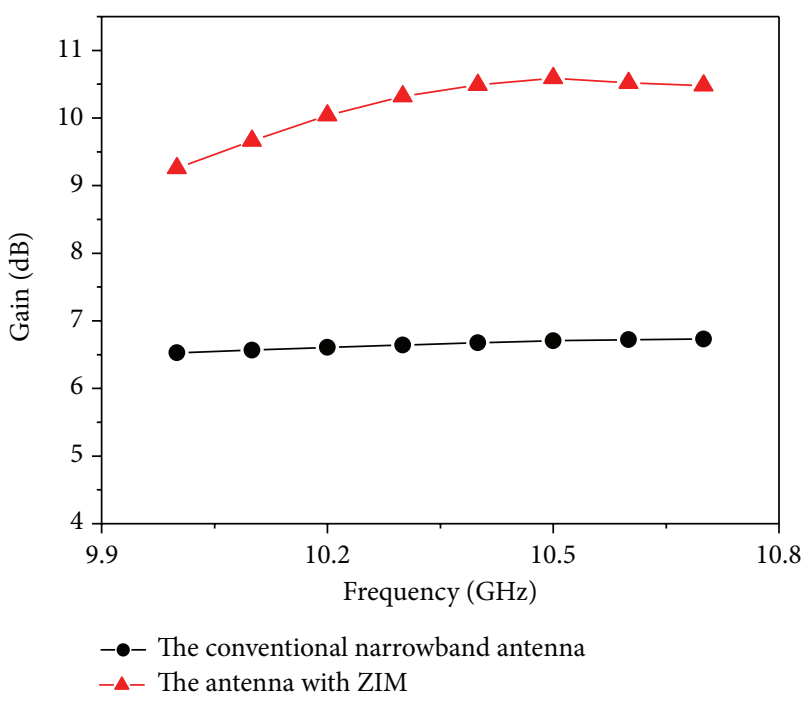

(a)

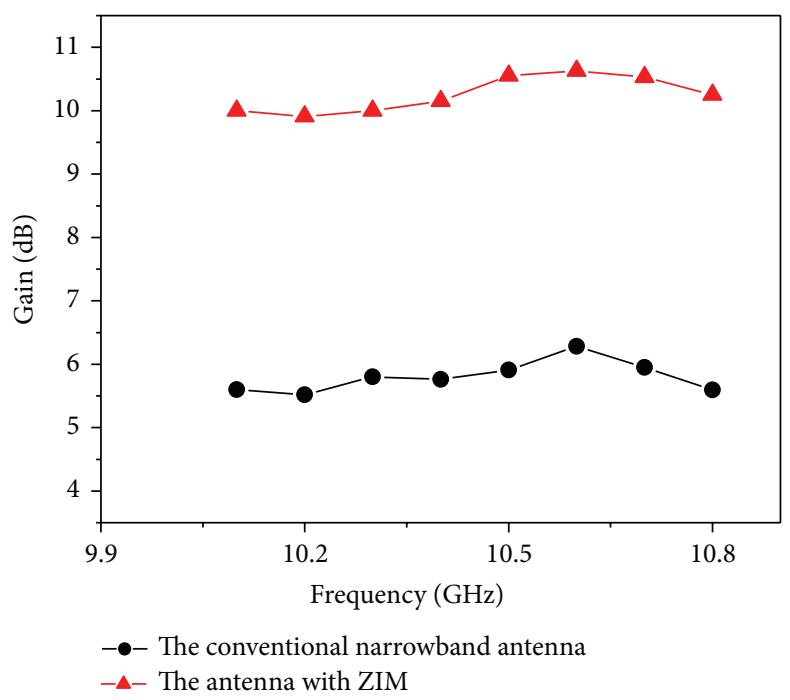

(b)

FIGURE 9: The comparative gains of the conventional narrowband antenna and the proposed antenna, (a) simulations and (b) measurements.

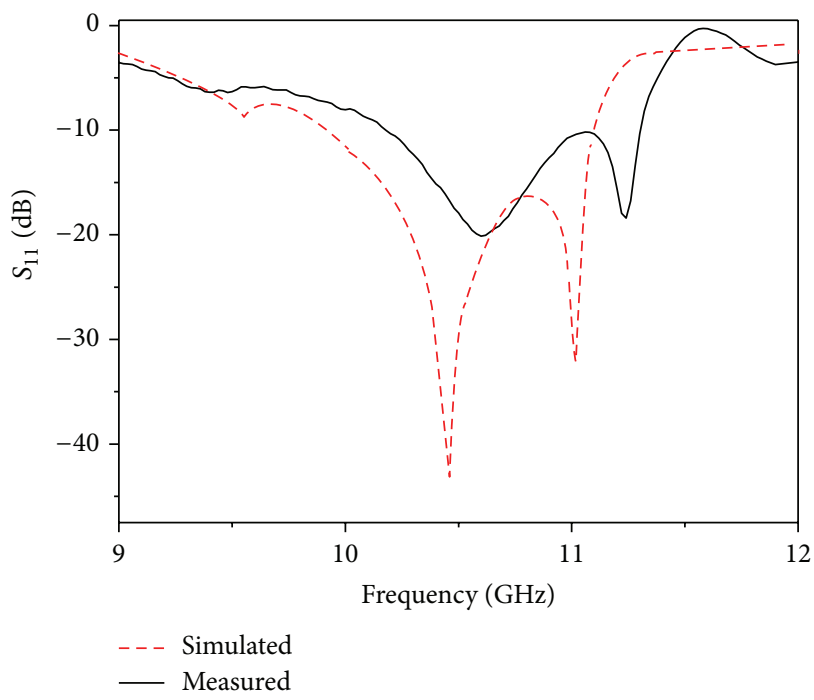

(a)

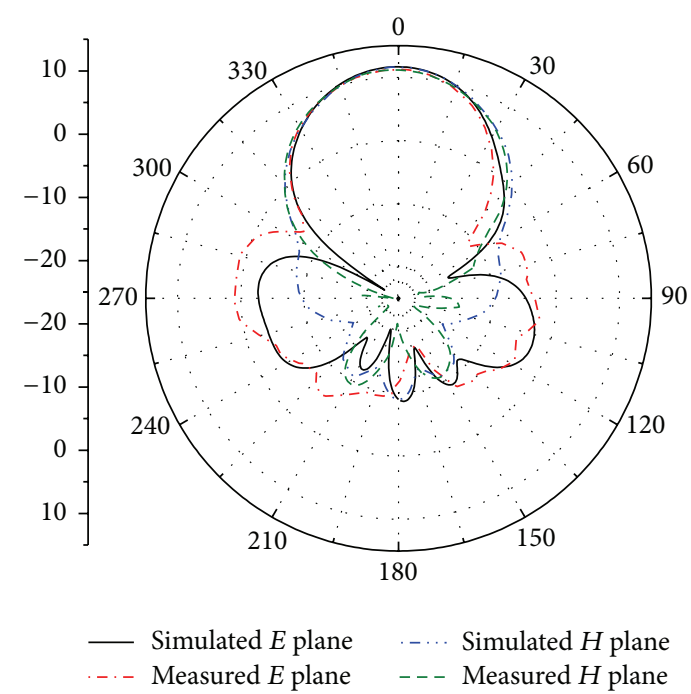

(b)

FIGURE 10: The performances of the proposed wideband antenna with the ZIM cover, (a) the reflection coefficients and (b) the radiation patterns.

To demonstrate the ZIM cover for improving antenna performance, the comparative radiation patterns between the conventional patch antenna and the proposed antenna are presented in Figure 8. It shows that the measured HPBW in the $E$ plane is reduced by $42^{\circ}$, and HPBW in the $H$ plane is reduced by $15^{\circ}$ compared to the conventional patch antenna without the ZIM. The side lobe is reduced and the forward radiation is enhanced. As a result, the gain is improved. The comparative gains are shows in Figure 9, which presents that the measured average gain is improved by $4.23 \mathrm{~dB}$ compared to the conventional antenna without the ZIM.
3.3. Performances of the Wideband Patch Antenna with the ZIM Cover. Figure 10 presents the performances of the proposed wideband antenna. The simulated and measured antenna reflection coefficients are shown in Figure 10(a), where the simulated $-10 \mathrm{~dB}$ bandwidth is $1.192 \mathrm{GHz}$ covering from $9.908 \mathrm{GHz}$ to $11.1 \mathrm{GHz}$ and the measured $-10 \mathrm{~dB}$ bandwidth is $1.18 \mathrm{GHz}$ covering from $10.14 \mathrm{GHz}$ to $11.32 \mathrm{GHz}$. The proposed antenna radiation patterns are shown in Figure 10(b). It shows that the simulated HPBW in the $E$ plane and $H$ plane are $36.4^{\circ}$ and $37.2^{\circ}$, respectively. The measured HPBW in the $E$ plane and $H$ plane are $38^{\circ}$ and 

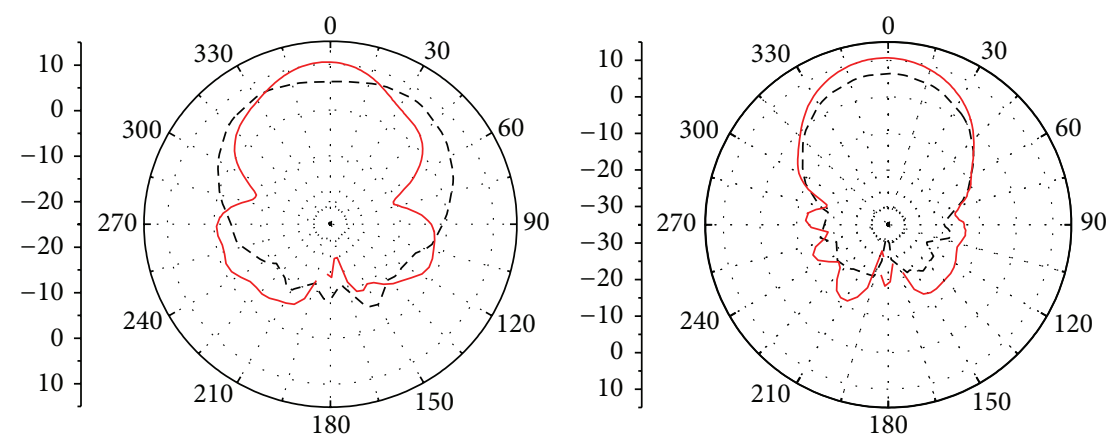

(a)
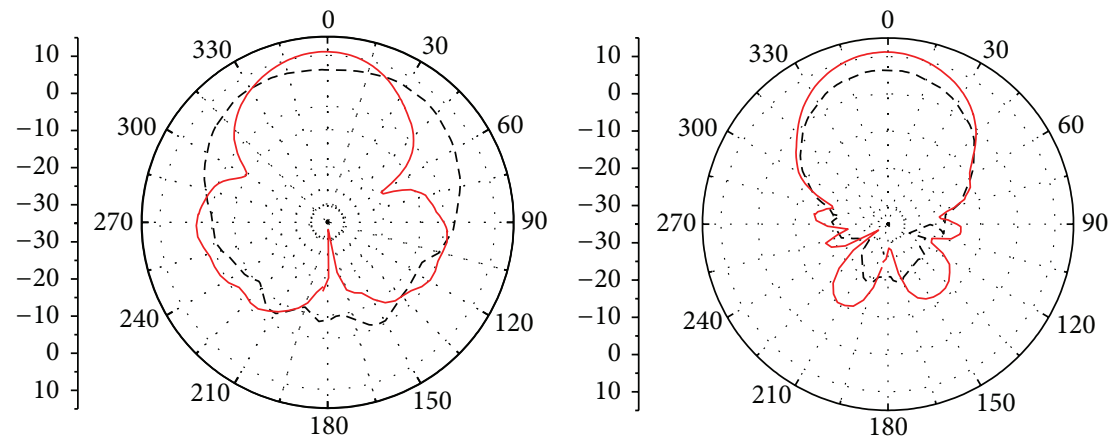

(b)
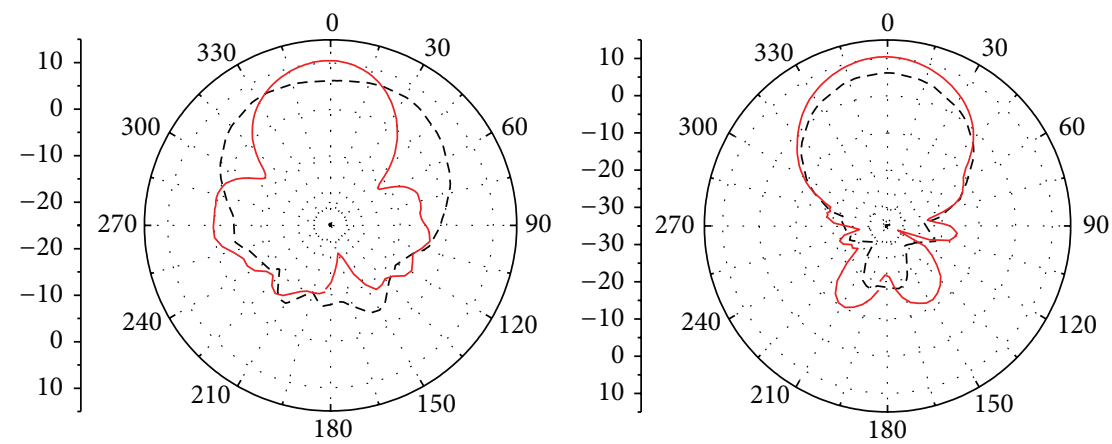

(c)
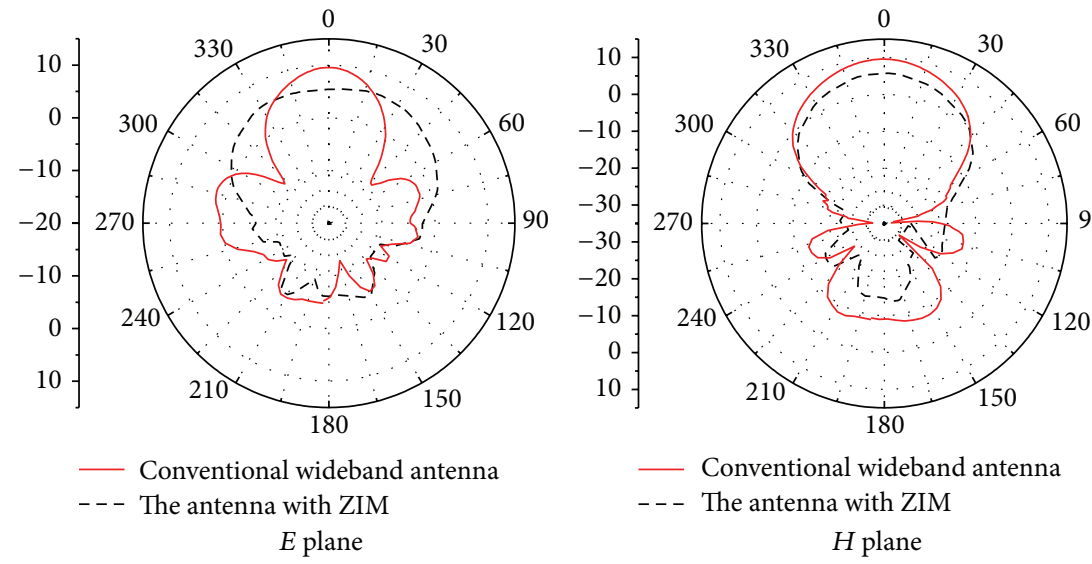

(d)

FiguRE 11: The measured radiation patterns for the conventional wideband antenna and the proposed antenna at the frequencies of (a) $10.15 \mathrm{GHz}$, (b) $10.6 \mathrm{GHz}$, (c) $10.8 \mathrm{GHz}$, and (d) $11.3 \mathrm{GHz}$. 


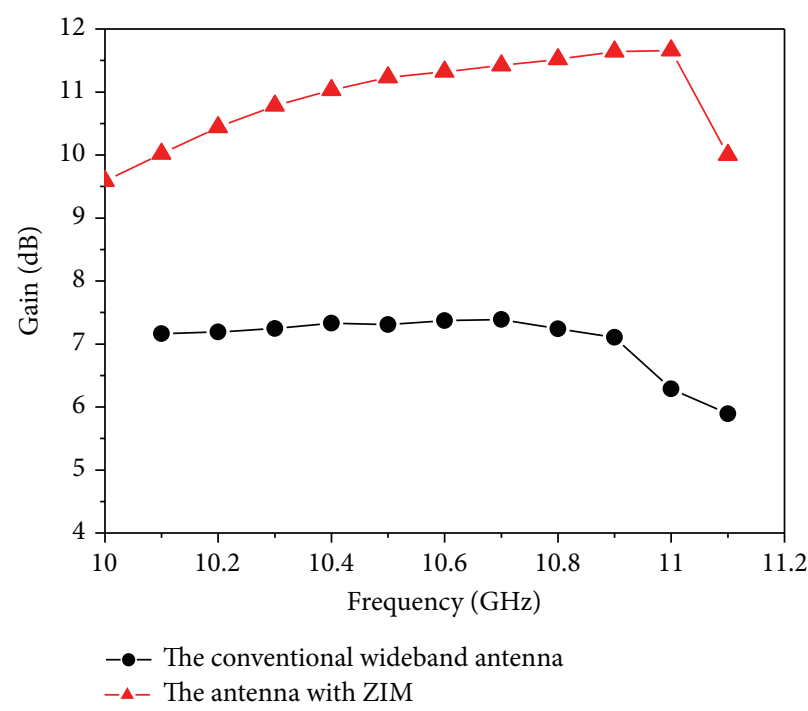

(a)



(b)

FIGURE 12: The comparative gains of the conventional wideband antenna and the proposed antenna, (a) simulations and (b) measurements.

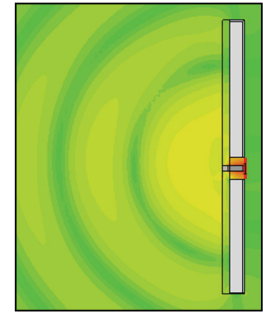

Without ZIM

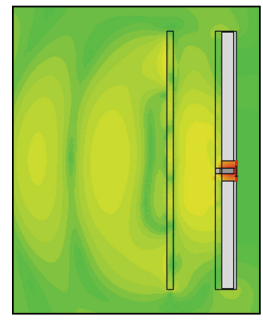

With ZIM

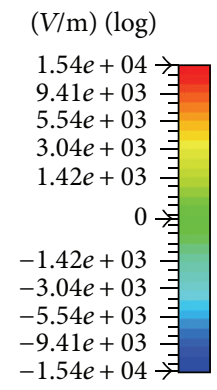

(a)

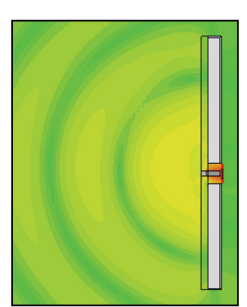

Without ZIM

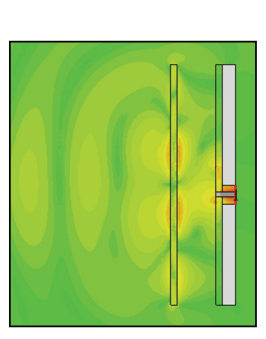

With ZIM

FIGURE 13: The comparative electric field magnitude distributions of the antennas, (a) $10 \mathrm{GHz}$ and (b) $11.1 \mathrm{GHz}$.

TABLE 1: The performances of the antenna based on the different ZIM layers.

\begin{tabular}{lccc}
\hline Antennas & \multicolumn{3}{c}{ Parameters } \\
& HPBW $\left(\theta_{E}\right)$ & HPBW $\left(\theta_{H}\right)$ & Gain $(\mathrm{dB})$ \\
\hline Patch antenna without ZIM & $106^{\circ}$ & $90^{\circ}$ & 6.643 \\
Antenna with 1 layer ZIM & $45^{\circ}$ & $51^{\circ}$ & 10.33 \\
Antenna with 2 layers ZIM & $35^{\circ}$ & $40^{\circ}$ & 10.8 \\
Antenna with 3 layers ZIM & $31^{\circ}$ & $36^{\circ}$ & 11.3 \\
Antenna with 4 layers ZIM & $28^{\circ}$ & $32^{\circ}$ & 12.4 \\
Antenna with 5 layers ZIM & $26^{\circ}$ & $28^{\circ}$ & 13.0 \\
Antenna with 6 layers ZIM & $23^{\circ}$ & $24^{\circ}$ & 13.7 \\
Antenna with 7 layers ZIM & $20^{\circ}$ & $20^{\circ}$ & 13.9 \\
\hline
\end{tabular}

$43^{\circ}$, respectively. The simulated gain is $11.63 \mathrm{~dB}$ and the measured one is $11.3 \mathrm{~dB}$ at the center frequency. The simulated and measured aperture efficiencies are $30.4 \%$ and $28.7 \%$, respectively.
The comparative antenna radiation patterns between the conventional wideband patch antenna and the proposed antenna are presented in Figure 11. The radiation patterns at the frequencies of $10.15 \mathrm{GHz}, 10.6 \mathrm{GHz}, 10.8 \mathrm{GHz}$, and $11.3 \mathrm{GHz}$ are all given in order to demonstrate that the antenna performs good performances at the wideband frequencies. It shows that HPBW in the $E$ plane is reduced by $49^{\circ}$ and HPBW in the $H$ plane is reduced by $22^{\circ}$ compared to the conventional wideband patch antenna without the ZIM cover. The comparative gains are presented in Figure 12, which shows that the measured average gain is improved by $4.37 \mathrm{~dB}$ compared to the conventional wideband antenna without the ZIM cover.

3.4. Discussion. In order to explore physically the improvement of antenna performance, simulated electric field distributions for the conventional wideband antenna and the antenna with the ZIM cover at the lower frequency $(10 \mathrm{GHz})$ and the upper frequency $(11.1 \mathrm{GHz})$ are given in Figure 13. The electromagnetic wave front presents a spherical wave for 
the conventional antenna without the ZIM cover at these two frequencies. However, the electromagnetic wave front shows a plane wave for the antenna with the ZIM cover. The planar ZIM cover plays a role in controlling the electromagnetic wave propagation direction, changing the spherical wave radiated by the conventional antenna to the plane wave. In the far-field view, the sideward radiation will be reduced, and forward radiation can be enhanced in the radiation patterns. As a result, a more directional and higher gain antenna can be obtained. The similar electric field distributions can be obtained for the conventional narrowband antenna and the antenna with the ZIM cover.

It is known that the propagation phase can be defined as $\Delta \phi=n k_{0} d$ when the electromagnetic wave transmits the distance $d$ in the medium. For the ZIM, the index is zero. Therefore, propagation phase is independent of propagation distance. It is expected that the propagation phase is the same at the interface between the medium and the free space whenever excitation of radiation source in the ZIM is the spherical wave or the plane wave. Hence, the form of the electromagnetic wave front depends on the curvature of the emergent surface when the electromagnetic wave transmits through the ZIM. In this paper, the planar ZIM can be employed for changing the spherical wave radiated by the conventional antenna to the plane wave. As a result, the directivity and gain of the antenna with the ZIM cover can be enhanced.

In the present paper, the high-gain patch antennas based on the ZIM cover are proposed. The planar ZIM structure in our paper is fabricated by using a single-layer shadow mask/etching microstrip technology, resulting in the merits of simple and planar structure, low profile, low weight, compact size, and easy fabrication. In addition, compared to the reported patch antennas $[25,26]$, our proposed patch antenna has the compact volume and a much better aperture efficiency. In summary, the proposed antenna has the advantages of more compact volume, better gain, and higher aperture efficiency. Hence, we provide a method to solve some limitations (low gain, low radiation efficiency) of the conventional patch antenna. It is regarded that using the planar ZIM to improve the gain of the conventional patch antenna is significant in this paper.

\section{Conclusions}

In this work, a wideband planar ZIM is investigated. According to the zero index, two high-gain patch antennas based on the ZIM cover are designed and fabricated. The optimal distance between the patch and the ZIM cover and the number of the ZIM layers are demonstrated. The antenna performances are studied with simulations and measurements. The results show that the energy radiated by the ZIM cover antennas becomes more concentrate. As a result, the more directional and higher gain antennas are obtained. The average gain for the narrowband proposed antenna is improved by $4.23 \mathrm{~dB}$. Besides the narrowband antenna, the antenna performance is improved at the wideband frequencies when the ZIM cover is placed above the wideband patch antenna.
The average gain for the proposed wideband antenna is improved by $4.37 \mathrm{~dB}$ as compared with the antenna without the ZIM cover.

It is significant that the wideband high-gain planar patch antenna based on the ZIM cover is realized. It is expected that the proposed high-gain antenna can be applied in the fields of high-rate data transmission, high-resolution radar systems, and among other fields. In addition, the ZIM has the merits of simple structure, compact size, and most importantly it can improve the antenna performances greatly at the wideband frequency. Furthermore, the planar ZIM cover can also be used with the other antennas such as monopoles, dipole antennas, leak-wave antennas, and aperture antennas.

\section{Acknowledgments}

This work is supported by the National Natural Science Foundation of China under Grants 11204241, 50936002, and 51272215, and by the NPU Foundation for Basic Research under Grants JC201154 and JC201153, and by the NPU Aoxiang Star Project.

\section{References}

[1] J. B. Pendry, A. J. Holden, W. J. Stewart, and I. Youngs, "Extremely low frequency plasmons in metallic mesostructures," Physical Review Letters, vol. 76, no. 25, pp. 4773-4776, 1996.

[2] J. B. Pendry, A. J. Holden, D. J. Robbins, and W. J. Stewart, "Magnetism from conductors and enhanced nonlinear phenomena," IEEE Transactions on Microwave Theory and Techniques, vol. 47, no. 11, pp. 2075-2084, 1999.

[3] H. S. Chen, L. X. Ran, J. T. Huangfu et al., "Left-handed materials composed of only S-shaped resonators," Physical Review E, vol. 70, no. 5, Article ID 057605, 2004.

[4] E. Kim, Y. R. Shen, W. Wu et al., "Modulation of negative index metamaterials in the near-IR range," Applied Physics Letters, vol. 91, no. 17, Article ID 173105, 2007.

[5] J. Zhou, L. Zhang, G. Tuttle, T. Koschny, and C. M. Soukoulis, "Negative index materials using simple short wire pairs," Physical Review B, vol. 73, no. 4, Article ID 041101, 2006.

[6] X. Zhou, Y. Liu, and X. P. Zhao, "Low losses left-handed materials with optimized electric and magnetic resonance," Applied Physics A, vol. 98, no. 3, pp. 643-649, 2010.

[7] H. T. Jiang, Z. L. Wang, Y. Sun et al., "Enhancement of (nearly) homogeneous fields in a (effective) zero-index cavity," Journal of Applied Physics, vol. 109, no. 7, Article ID 073113, 2011.

[8] Y. Jin and S. He, "Enhancing and suppressing radiation with some permeability-near-zero structures," Optics Express, vol. 18, no. 16, pp. 16587-16593, 2010.

[9] M. Silveirinha and N. Engheta, "Tunneling of electromagnetic energy through subwavelength channels and bends using $\mathcal{E}$ near-zero materials," Physical Review Letters, vol. 97, no. 15, Article ID 157403, 2006.

[10] R. Liu, Q. Cheng, T. Hand et al., "Experimental demonstration of electromagnetic tunneling through an epsilon-nearzero metamaterial at microwave frequencies," Physical Review Letters, vol. 100, no. 2, Article ID 023903, 2008. 
[11] B. Edwards, A. Alù, M. E. Young, M. Silveirinha, and N. Engheta, "Experimental verification of epsilon-near-zero metamaterial coupling and energy squeezing using a microwave waveguide," Physical Review Letters, vol. 100, no. 3, Article ID 033903, 2008.

[12] Y. Jin, P. Zhang, and S. He, "Squeezing electromagnetic energy with a dielectric split ring inside a permeability-near-zero metamaterial," Physical Review B, vol. 81, no. 8, Article ID 085117, 2010.

[13] V. C. Nguyen, L. Chen, and K. Halterman, "Total transmission and total reflection by zero index metamaterials with defects," Physical Review Letters, vol. 105, no. 23, Article ID 233908, 2010.

[14] J. Hao, W. Yan, and M. Qiu, "Super-reflection and cloaking based on zero index metamaterial," Applied Physics Letters, vol. 96, no. 10, Article ID 101109, 2010.

[15] S. Enoch, G. Tayeb, P. Sabouroux, N. Guérin, and P. Vincent, "A metamaterial for directive emission," Physical Review Letters, vol. 89, no. 21, Article ID 213902, 2002.

[16] B. I. Wu, W. Wang, J. Pacheco, X. Chen, and J. A. Kong, "A study of using metamaterials as antennasubstrate to enhance gain," Progress in Electromagnetics Research, vol. 51, pp. 295-328, 2005.

[17] Z. B. Weng, Y. Song, Y. C. Jiao, and F. S. Zhang, "A directive dual-band and dual-polarized antenna with zero index metamaterial," Microwave and Optical Technology Letters, vol. 50, no. 11, pp. 2902-2904, 2008.

[18] H. Zhou, Z. Pei, S. Qu et al., "A novel high-directivity microstrip patch antenna based on zero-index metamaterial," IEEE Antennas and Wireless Propagation Letters, vol. 8, pp. 538-541, 2009.

[19] Y. L. Lv, F. Y. Meng, J. Hua, and M. L. Chen, "A wideband zero index metamaterial lens for directive emission based on $\mathrm{Z}$ shaped meta-atom," in Proceedings of the 5th Global Symposium on Millimeter Waves (GSMM '12), pp. 418-421, 2012.

[20] Q. W. Hou, H. F. Tang, Y. H. Liu, and X. P. Zhao, "Dualfrequency and broadband circular patch antennas with a monopole-type pattern based on epsilon-negative transmission line," IEEE Antennas and Wireless Propagation Letters, vol. 11, pp. 442445, 2012.

[21] Y. H. Liu, S. Gu, C. R. Luo, and X. P. Zhao, "Ultra-thin broadband metamaterial absorber," Applied Physics A, vol. 108, pp. 1924, 2012.

[22] Y. Yao, X. Wang, and J. Yu, "Multiband planar monopole antenna for LTE MIMO systems," International Journal of Antennas and Propagation, vol. 2012, Article ID 890705, 6 pages, 2012.

[23] D. R. Smith, D. C. Vier, T. Koschny, and C. M. Soukoulis, "Electromagnetic parameter retrieval from inhomogeneous metamaterials," Physical Review E, vol. 71, no. 3, Article ID 036617, 2005.

[24] R. Garg, R. Bhartia, I. Bahl, and A. Ittipiboon, Microstrip Antenna Design Handbook, Artech House, Boston, Mass, USA, 2001.

[25] A. P. Feresidis, G. Goussetis, S. Wang, and J. C. Vardaxoglou, "Artificial magnetic conductor surfaces and their application to low-profile high-gain planar antennas," IEEE Transactions on Antennas and Propagation, vol. 53, no. 1 I, pp. 209-215, 2005.

[26] F. M. Zhu, Q. C. Lin, S. L. He, J. Hu, and Z. N. Ying, "A high directivity patch antenna using a PBG cover together with a PBG substrate," in Proceeding of the 6th International Symposium on Antennas, Propagation and EM Theory, pp. 9295, November 2003. 

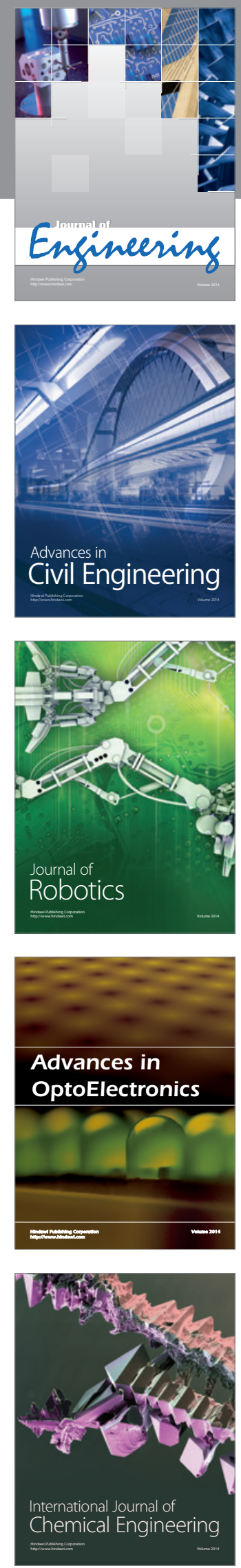

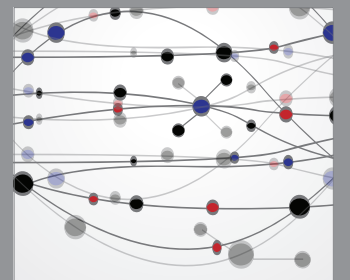

The Scientific World Journal
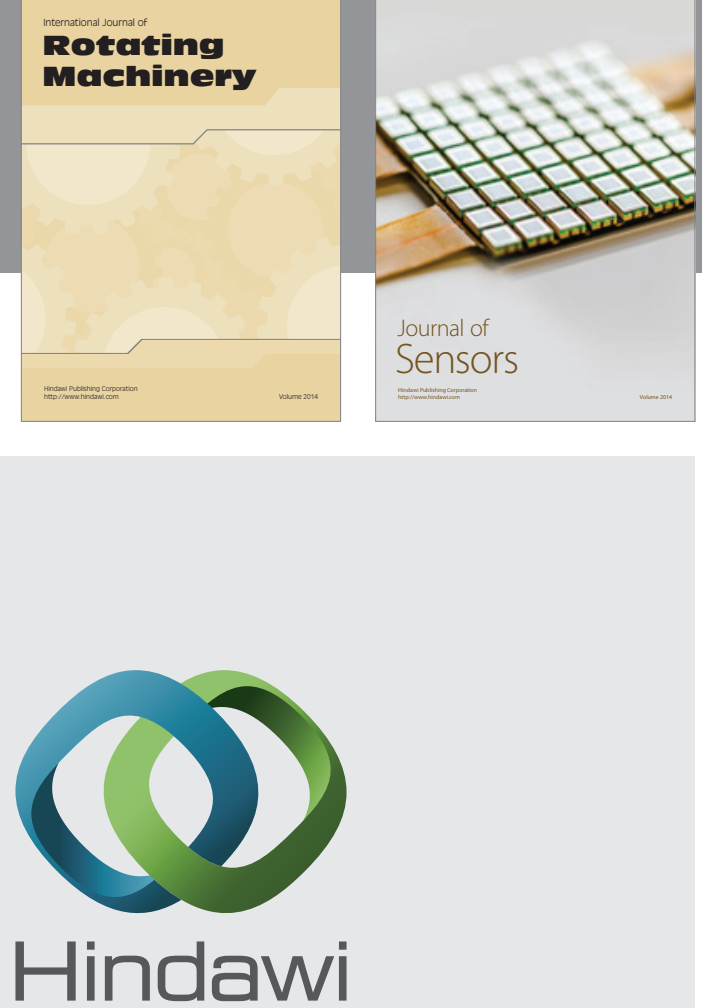

Submit your manuscripts at http://www.hindawi.com
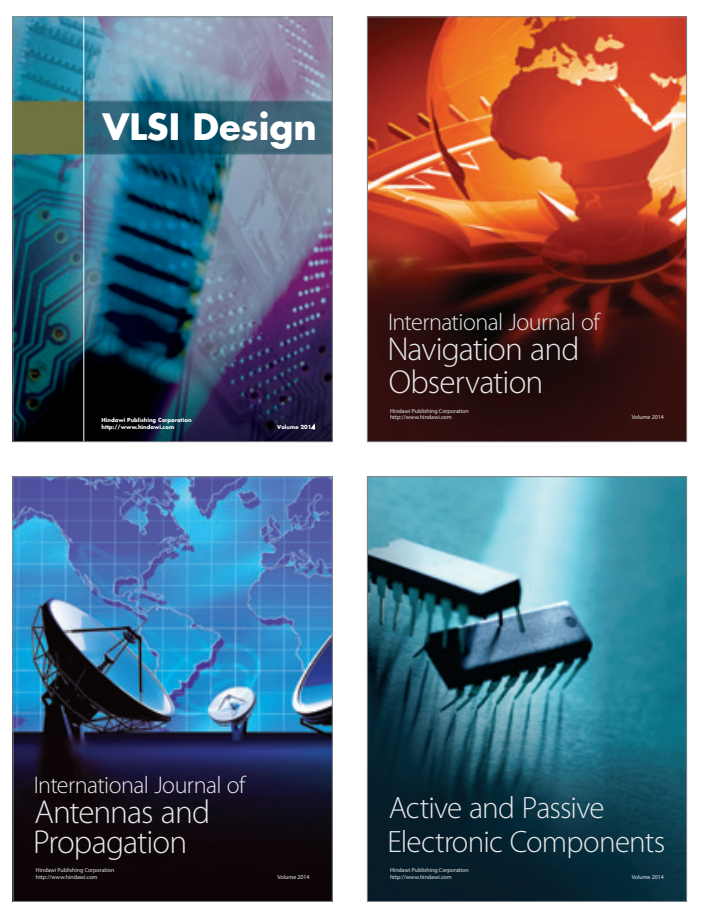
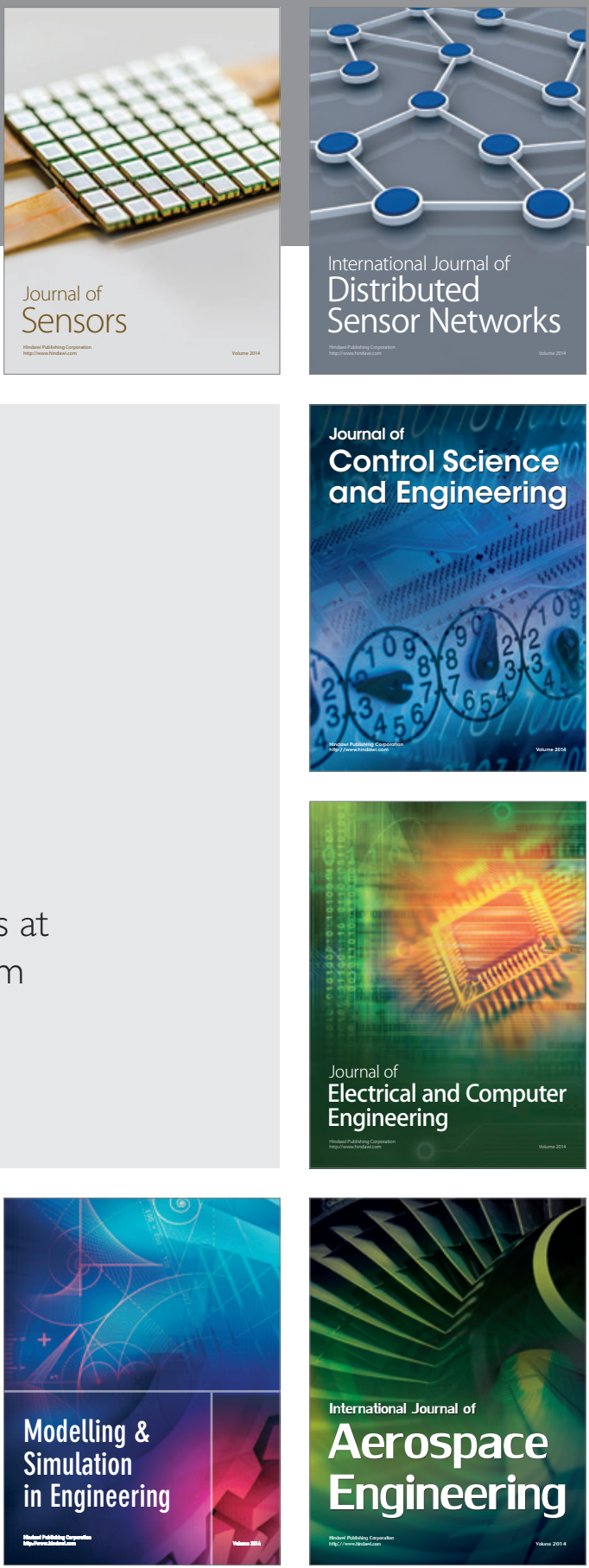

Journal of

Control Science

and Engineering
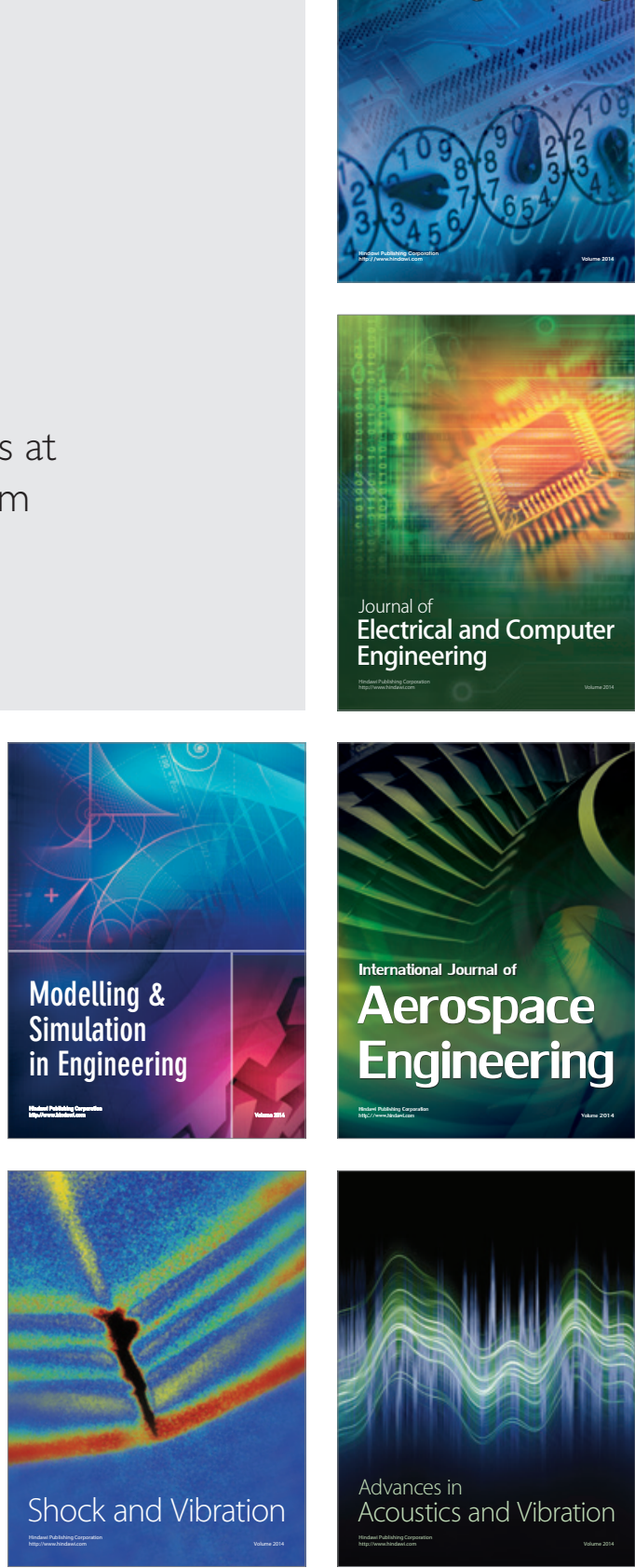